\title{
Use of random noise for on-line transducer modeling in an adaptive active attenuation system ${ }^{\text {a) }}$
}

\author{
L.J. Eriksson and M. C. Allie \\ Corporate Research Department, Nelson Industries, Inc., P. O. Box 600, Stoughton, Wisconsin 53589-0600
}

(Received 9 January 1987; accepted for publication 26 October 1988)

\begin{abstract}
Active sound attenuation systems may be described using a system identification framework in which an adaptive filter is used to model the performance of an unknown acoustical plant. An error signal may be obtained from a location following an acoustical summing junction where the undesired noise is combined with the output of a secondary sound source. For the model output to properly converge to a value that will minimize the error signal, it is frequently necessary to determine the transfer function of the secondary sound source and the path to the error signal measurement. Since these transfer functions are unknown and continuously changing in a real system, it is desirable to perform continuous on-line modeling of the output transducer and error path. In this article, the use of an auxiliary random noise generator for this modeling is described. Based on a Galois sequence, this technique is easy to implement, provides continuous on-line modeling, and has minimal effect on the final value of the error signal.
\end{abstract}

PACS numbers: 43.60.Gk, 43.50.Ki

\section{INTRODUCTION}

Active sound attenuation is a relatively old idea that has received considerable attention in recent years. This is primarily due to the development of improved signal-processing theory and hardware that enable more sophisticated approaches to this problem. Many of the traditional problems with this technology can now be treated more effectively with proper signal processing rather than with the direct acoustical approaches of the past.

This article describes a complete active attenuation system that functions correctly in the presence of acoustic feedback as well as nonideal input microphone, error microphone, loudspeaker, and error path transfer functions. It is completely adaptive and responds automatically to changes in input signal, acoustic plant, error plant, microphone, and loudspeaker characteristics.

\section{SYSTEM IDENTIFICATION}

Active sound attenuation systems may be described using a system identification framework in which an adaptive filter is used to model the performance of an unknown acoustical plant, as shown in Fig. 1 (a)..$^{1-5}$ An input microphone is used to measure the undesired noise upstream of the acoustical plant. This signal is used as the input to an adaptive filter that generates an output to a loudspeaker which is used to produce a secondary sound that is acoustically combined with the undesired noise. An error signal measured downstream from the acoustical summing junction is used to adapt the coefficients of the adaptive filter to minimize the residual noise. When fully adapted, the adaptive filter response in series with the response of the input microphone and loudspeaker matches the response of the acoustical plant.

\footnotetext{
- An earlier version of this article was presented at the 112th Meeting of the Acoustical Society of America, 8-12 December 1986 in Anaheim, CA [J. Acoust. Soc. Am. Suppl. 1 80, S11 (1986)].
}

Although this system identification problem has been intensively studied in the control and signal-processing literature, the active attenuation application is complicated by the presence of acoustic feedback from the loudspeaker to the input microphone. In the past, a variety of solutions to this problem have been proposed that utilize either directional transducer arrays or incorporate a compensating fixed

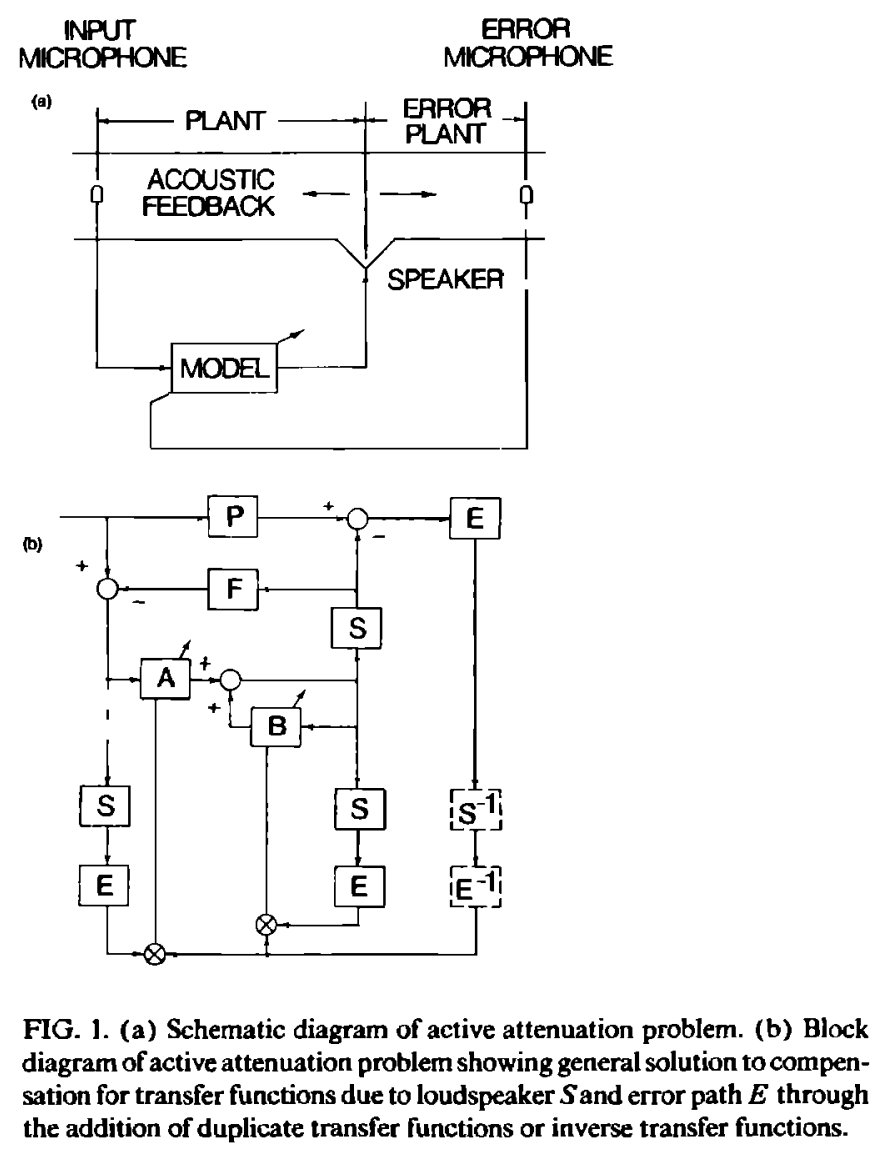


feedback path that is determined on an off-line basis through calculations or use of a training signal.

Eriksson has presented a new technique for active attenuation that effectively utilizes adaptive signal processing to solve the problem of acoustic feedback from the secondary sound source to the input microphone. ${ }^{6}$ This technique utilizes a recursive-least-mean-squares (RLMS) algorithm developed by Feintuch ${ }^{7}$ to provide a complete pole-zero model of the acoustical plant. The acoustic feedback is considered part of the adaptive model used to model the plant. From this perspective, the acoustic feedback introduces fixed poles into the overall response of the model, which may be removed with the pole-zero response of the RLMS algorithm. Using the configuration shown in Fig. 1(b), the direct acoustical path $P$ and feedback acoustical path $F$ are simultaneously modeled by the RLMS model using adaptive filters $A$ and $B$, in series with the loudspeaker $S$. Perfect cancellation is obtained when the overall model response matches the response of the plant or using $z$ transforms:

$$
M_{T}=M S /(1+F M S)=A S /(1-B+F A S)=P,
$$

where

$\boldsymbol{M}_{T}=$ overall model response,

$\boldsymbol{M}=$ response of pole-zero recursive filter structure used in RLMS algorithm,

$A=$ response of all-zero least-mean-squares (LMS) element used in direct path of pole-zero structure,

$B=$ response of all-zero LMS element used in recursive path of pole-zero structure,

$P=$ direct path acoustic plant,

$F=$ feedback path acoustic plant, and

$S=$ response of loudspeaker.

One solution to this equation is for $A=P / S$ and $B=P F$. However, the actual model response is a complex function of the spectral content of the source and the acoustical plant of the system. The RLMS algorithm provides a fully adaptive means to simultaneously model the direct plant and feedback plant with a given source in such a way as to minimize the residual noise.

\section{TRANSDUCER MODELING}

One of the problems with this technique is that the RLMS algorithm requires knowledge of the speaker transfer function and error path transfer function for proper convergence. ${ }^{1}$ Widrow ${ }^{8}$ has shown that the LMS algorithm can be used with a delayed error signal if the input to the error correlators is also delayed by the same amount. Similarly, Morgan has stated that, with proper compensation, the LMS algorithm can also be used if a transfer function, such as that due to the loudspeaker, is in the auxiliary path following the adaptive filter. Proper compensation requires the addition of a transfer function in the input to the error correlators or the addition of an inverse transfer function in series with the error path. ${ }^{9}$ Burgess has discussed similar results for the
LMS algorithm when both auxiliary path and error path transfer functions are present. ${ }^{10} \mathrm{~A}$ transfer function is added to the input to the error correlators, which represents the product of the auxiliary path and error path transfer functions. Widrow and Stearns ${ }^{11,12}$ have similarly discussed the "filtered- $X$ " LMS algorithm for use with a plant in the auxiliary path. ${ }^{11,12}$

These results have been extended to an infinite impulse response (IIR) adaptive filter using the RLMS algorithm by Eriksson. ${ }^{1}$ The speaker transfer function $S$ and error path transfer function $E$ must be known to compensate for their effect on the convergence of both the direct and recursive elements of the IIR filter. This can be done through either the addition of $S$ and $E$ into the input lines to the error correlators or the addition of the inverse transfer functions, $S^{-1}$ and $E^{-1}$, into the error path, as shown in Fig. 1(b). As discussed above, the former technique has been described by Widrow $^{8}$ and Burgess ${ }^{10}$ for the LMS algorithm and assures that the error signal and input signal will have the same relationship in time. The latter technique has been described by Morgan ${ }^{9}$ for the LMS algorithm and eliminates the need for a modification to the input signal in principle, but, in practice, the lack of causality for the inverse transfer functions, $S^{-1}$ and $E^{-1}$, requires compensation of the input signal to the error correlators by delay, as will be discussed in the following.

Unfortunately, both $S$ and $E$ are unknown and are time varying due to effects such as heat and aging on the loudspeaker and due to changes in temperature and flow in the error path. Thus it is necessary to obtain either direct or inverse models of $S$ and $E$ on an on-line basis. Although they are not shown explicitly in Fig. 1(b), the error microphone may be considered as part of the error path transfer function $E$, and the input microphone simply adds an additional transfer function in series with the RLMS model and loudspeaker $S$. Since the input microphone occurs prior to the adaptive model, it does not need to be compensated for in the same manner as $S$ and $E$.

Poole et al. ${ }^{13}$ have described a system using the LMS algorithm in which a fixed compensating inverse transfer function is added to the error path. However, since $S^{-1}$ and $E^{-1}$ are noncausal, an off-line model of a delayed inverse model of the loudspeaker and error path $\Delta S^{-1} E^{-1}$ is determined where $\Delta$ is the delay necessary to make the inverse model causal. ${ }^{14}$ The use of this delayed inverse model reduces the error path transfer function to a fixed pure delay $\Delta$. As noted above, this approach then requires the addition of the same delay $\Delta$ to the input to the error correlators of the LMS algorithm as described by Widrow. ${ }^{8}$ The primary disadvantage of this technique is that it does not use an on-line, continuously adaptive model of the loudspeaker and error path.

Eriksson ${ }^{1}$ has described a three-microphone system using the RLMS algorithm in which the error plant is modeled on line using either a direct or inverse model while the speaker is modeled off line. However, there have not been any previous approaches described that provide an on-line model of the speaker and the error path that responds to changes in their response over time. 


\section{MODELING APPROACHES}

There are two basic techniques available for use in system modeling using adaptive filters. The direct approach places the model in parallel with the unknown plant and is adapted such that the difference between the outputs of the plant and model is minimized for the same signal. The inverse approach places the model in series with the unknown plant such that the difference between the output of this series combination and a delayed version of the input signal is minimized. In this case, the response of the adaptive model becomes a delayed version of the inverse of the unknown plant response.

As shown in Fig. 2(a), to determine the speaker and error path response, the direct model approach places the adaptive model in parallel with the speaker and error path. An error signal formed by subtracting the adaptive model output from the microphone output is multiplied by the input signal to form the update terms for the coefficients of the adaptive model. The inverse model approach places the adaptive model in series with the speaker and error path, as shown in Fig. 2(b). In this case, the error signal formed by subtracting the adaptive model output from a delayed version of the noise input is multiplied by the input to the adaptive model to form the update terms for the coefficients of the adaptive model. Thus the adaptive model forms a delayed inverse model of the speaker and the error path while attempting to match the response of the delayed noise input.
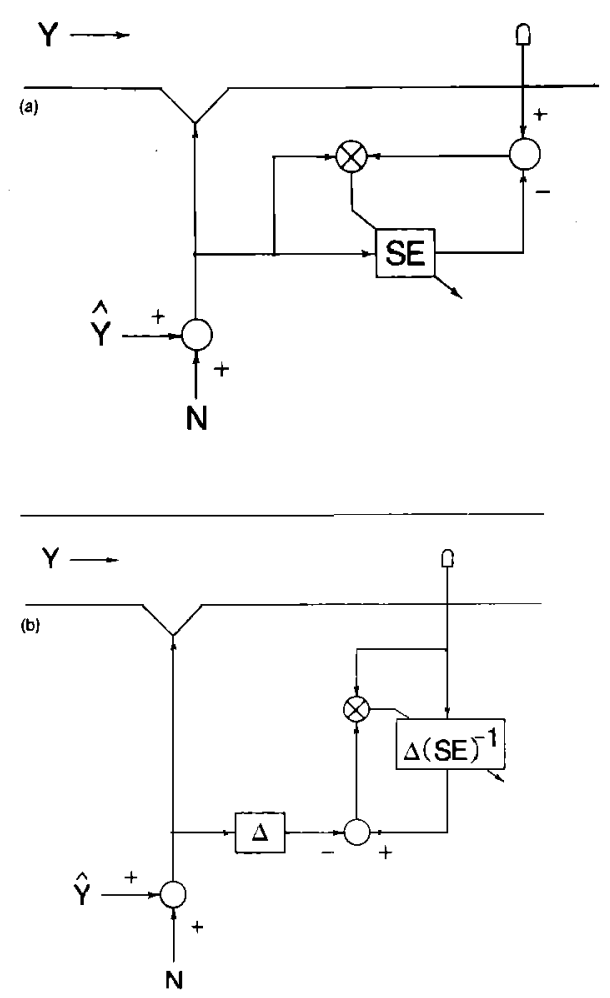

FIG. 2. (a) Direct modeling approach for the determination of the transfer function of the speaker and error path with adaptive model $S E$. (b) Inverse modeling approach for the determination of the delayed inverse transfer function of the speaker and error path with adaptive model $\Delta(S E)^{-1}$.
The traditional solution is then to use either the direct or inverse modeling approach shown in Fig. 2(a) and (b), respectively, on an off-line basis with a broadband noise source $N$. Since it is an off-line process, the plant output $y$ and model output $\hat{y}$ are not present. The noise source $N$ thus allows a precise determination of either the speaker and error path response, $S E$, or the delayed inverse model of the speaker and error path, $\Delta S^{-1} E^{-1}$. In the direct approach of Fig. 2(a), the response $S E$ is fixed after convergence and then used in the inputs to the error correlators of the LMS or RLMS algorithms. In the inverse approach of Fig. 2(b), the response $\Delta S^{-1} E^{-1}$ is also fixed after convergence, and the modeling delay $\Delta$ is used in the inputs to the error correlators of the LMS or RLMS algorithms. Both techniques assume the use of a large-amplitude, broadband noise source on an off-line basis to avoid contamination of the modeling process by $y$ or $\hat{y}$ and to avoid the addition of undesired noise during on-line operation by the noise source $N$.

\section{CONTINUOUS MODELING SYSTEM}

A new approach to the on-line modeling of $S$ and $E$ is shown in Fig. 3. An uncorrelated random noise source is used to excite the series combination of the speaker followed by the error plant as well as adaptive model $C$ while the system is operating. ${ }^{15}$ This random noise source will ultimately become the source of the residual noise of the system. The direct adaptive model $C$ is used to obtain coefficients describing the response of $S$ and $E$ that can be used in the input lines to the error correlators for the primary RLMS algorithm. The generalized model output and weight update equations for the recursive adaptive filter may be written following the notation of Widrow and Stearns ${ }^{12}$ as

$$
\begin{aligned}
& y_{k}=W_{k}^{T} U_{k}, \\
& W_{k+1}=W_{k}+2 M U_{k}^{\prime} e_{k}, \\
& U_{k}^{\prime}=\left[u_{k}^{\prime}, u_{k-1}^{\prime}, \ldots\right]^{T}, \\
& u_{k}^{\prime}=C_{k}^{T} U_{k},
\end{aligned}
$$

where

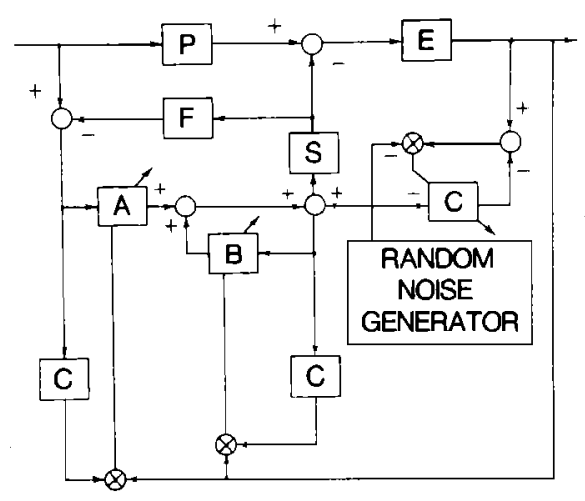

FIG. 3. New approach to on-line modeling of speaker $S$ and error path $E$ and using results in RLMS model with acoustic feedback to form a fully adaptive active attenuation system. 
$y_{k}=$ scalar model output at discrete time $k$,

$W_{k}=$ generalized weight vector (includes direct and recursive coefficients),

$W_{k}^{T}=$ transpose of $W_{k}$,

$W_{k+1}=$ updated generalized weight vector,

$\boldsymbol{M}=$ convergence factor matrix,

$e_{k}=$ scalar error signal,

$U_{k}=$ generalized input vector (includes direct and recursive input vectors),

$U_{k}^{\prime}=$ compensated generalized input vector,

$u_{k}^{\prime}=$ first component of compensated input vector, and

$C_{k}^{r}=$ transpose of weight vector of model associated with transfer functions in auxiliary path.

The weight vector of the adaptive model $C$ is obtained on an on-line basis using an adaptive algorithm such as the LMS or RLMS algorithm with the independent random noise source as an input and the error signal as shown in Fig. 3. The amplitude of the noise source is kept very low so that the final effect on the residual noise is small. The plant noise $y$ and model output $\hat{y}$ are not present at the input to the adaptive model $C$ and so will not affect the final values of the model weights.

The use of an uncorrelated random noise source that is independent of the input signal ensures that the speaker and error path will be correctly modeled. The signals from the plant $(y)$ and model $(\hat{y})$ represent noise on the "plant" side of the speaker/error path modeling process that will not affect the weights of the direct model $C$ used to determine $S E .^{12}$ This model is then copied to the input lines of the error correlators of the RLMS algorithm.

It should be noted that, although the delayed adaptive inverse model shown in Fig. 2(b) could be used in a similar fashion, this will result in decreased performance since the "noise" in the auxiliary path and error path due to $y$ and $\hat{y}$ also appears at the input of the adaptive filter due to the series arrangement. Thus the autocorrelation function of the filter input is adversely affected, and the filter weights are modified as described by Widrow and Stearns. ${ }^{12}$ If this "noise" is large enough, the adaptive model may fail to converge. Thus the delayed adaptive inverse approach requires a much larger amplitude random noise source that increases the residual noise and decreases overall system quieting.

In the direct model system, shown in Fig. 3, the "noise" due to $y$ and $\hat{y}$ does not affect the final weights in the adaptive model. In addition, the convergence of the $S E$ model is assured as long as the initial amplitudes are within the dynamic range and signal-to-noise ratio constraints of the system. Thus, with $S E$ accurately determined, the overall system model will converge, resulting in minimum residual noise.

The random noise source used to model $S E$ may be readily obtained through the use of a variety of methods. One simple approach is to generate a Galois sequence using methods described by Schroeder. ${ }^{16} \mathrm{~A}$ Galois sequence is a pseudorandom sequence that repeats after $2^{m}-1$ points, where $m$ is the number of stages in a shift register. It is easy to calculate and can easily have a period much longer than the response time of the system. In this study, 31 stages $(m=31)$ were used.

\section{RESULTS}

The results of a computer simulation of the system shown in Fig. 3 confirmed that the algorithm properly converges for either narrow-band or broadband input signals. The coefficients of the $S E$ model properly describe the $S E$ plant, and the coefficients of the overall system model properly describe $P, F$, and $S$.

The approach shown in Fig. 3 has also been implemented on complete acoustical systems using the TMS320 family of digital signal processing microprocessors, with input microphones, canceling loudspeakers, and error microphones. ${ }^{1.17 .18}$ Initially, one of these systems was utilized to cancel electroacoustically generated noise in a 12-in.-diam circular duct. The duct was about $25 \mathrm{ft}$ long and unlined except for a short 4-ft-long adsorptive silencer near the primary noise source. Typical results after adaptation are shown in Fig. 4. The noise reduction obtained with the system operating for a broadband noise input is shown in Fig. $4(a)$. This curve was obtained by subtracting the canceled spectrum from the uncanceled spectrum. The maxima and minima in the spectrum are due to acoustical resonances. The converged weight structure for the $A, B$, and $C$ elements of Fig. 3 is shown in Fig. 4(b). The decay of the coefficients confirms that the filter length chosen was adequate. The system is effective on broadband as well as narrow-band noise and requires no calibration or training of any kind.

Performance in an actual industrial fan or heating, ventilating, and air conditioning duct is made much more difficult by the turbulent airflow and large duct dimensions that are usually required. Good system performance requires antiturbulence microphones as well as large, powerful, lowfrequency sources. ${ }^{19}$ Typical results obtained are shown in Fig. 5. The uncanceled autospectrum in a lined supply duct ( $34 \times 44 \mathrm{in}$.) approximately $40 \mathrm{ft}$ from a centrifugal fan is

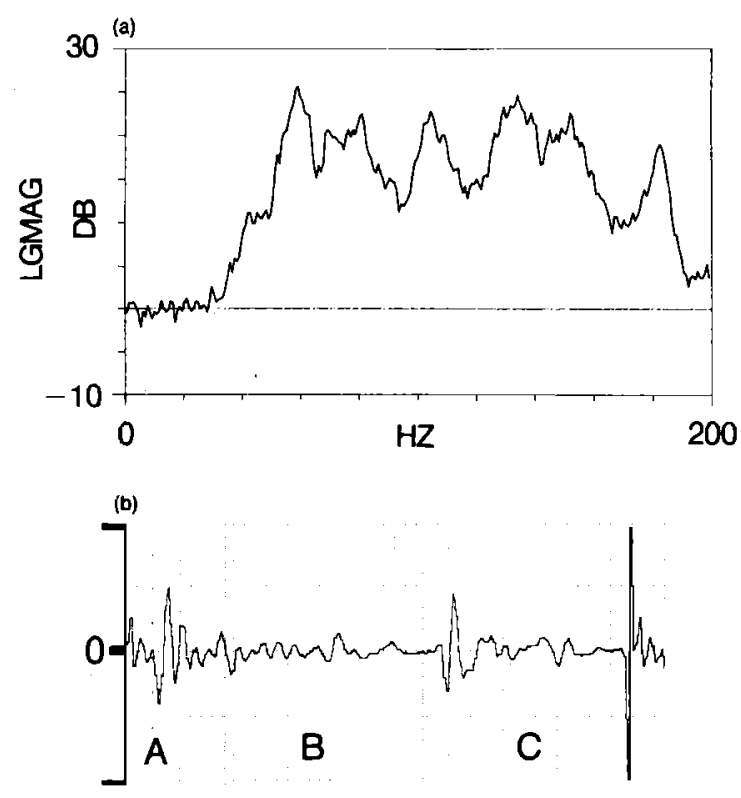

FIG. 4. (a) Noise reduction with active attenuation system on for bandlimited (15-200 Hz) pink noise input signal (no flow-128 averages). (b) Filter coefficients used to obtain the results shown in (a) for adaptive filters $A$ (32 taps), $B$ (64 taps), and $C$ (64 taps). 

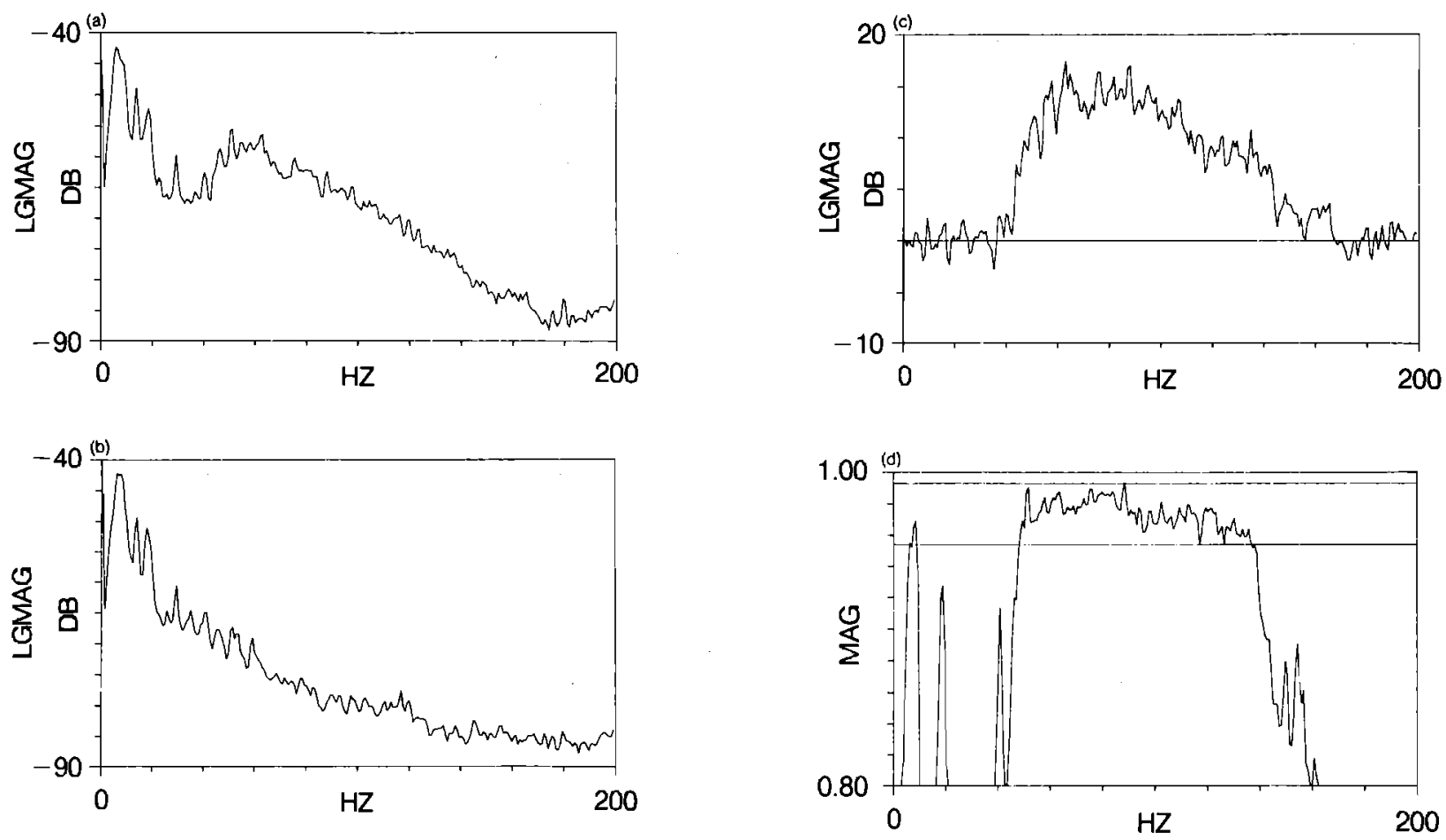

FIG. 5. (a) Relative sound-pressure spectrum in discharge duct of centrifugal fan with active attenuation system off [ Mach number ( $M$ ) $=0.04-128$ averages]. (b) Relative sound-pressure spectrum in discharge duct of centrifugal fan with active attenuation system on ( $M=0.04-128$ averages). (c) Noise reduction obtained from Fig. 5(a) and (b). (d) Typical coherence between input microphone and error microphone before the cancellation used to obtain the results of Fig. 5(c) ( $M=0.04-128$ averages).

shown in Fig. 5(a). In addition to a very-low-frequency peak at about $8 \mathrm{~Hz}$, there is a broad peak of noise from about 40 $\mathrm{Hz}$ to about $140 \mathrm{~Hz}$. With the active sound control system, this broad peak was reduced, as shown in Fig. 5(b). The noise reduction is plotted in Fig. $5(\mathrm{c})$. There is a broad range of attenuation from about 40 to $140 \mathrm{~Hz}$ peaking at about 18 dB. System performance is limited by the effectiveness of the antiturbulence microphones. There is minimal attenuation in the 8- to 40- $\mathrm{Hz}$ range due to the lack of coherence between the input and error microphones at these frequencies, as shown in Fig. 5(d). It should be noted that it has been found that the magnitude of the coherence must be on the order of 0.95 or greater for cancellation to be effective. The excellent coherence from about $40-140 \mathrm{~Hz}$ is consistent with the attenuation shown in Fig. 5(c). It would be difficult to obtain this performance using a conventional passive silencer. In addition, the active attenuation system results in essentially no restriction to the flow, thus avoiding the need to modify the fan drive. As before, the system is fully adaptive, and the results shown were obtained with no training or calibration before operating the system.

To demonstrate the effectiveness of the system on narrow-band as well as broadband noise, an electronic tone generator was used with a loudspeaker to introduce a tone at about $70 \mathrm{~Hz}$ at the fan while the HVAC system was operating. The noise reduction on this combined broadband fan noise and narrow-band electronic tone is shown in Fig. 6. The tone is reduced about $30 \mathrm{~dB}$, while the broadband noise is reduced about $15 \mathrm{~dB}$. It is important to note also that, in addition to the attenuation shown in Figs. 5(c) and 6, there is essentially no noise added at any frequency. Additional performance results have been presented elsewhere. ${ }^{3,20}$

Although these results are substantially better than typical passive silencers, there do not appear to be any reasons why the performance of this system cannot be increased. The primary areas of potential improvement are to obtain better coherence between the input and error microphones through the use of improved antiturbulence microphones and to increase the speed of computation through the use of more powerful microprocessors.

\section{CONCLUSIONS}

A complete active attenuation system has been described in which acoustic feedback is modeled as part of an

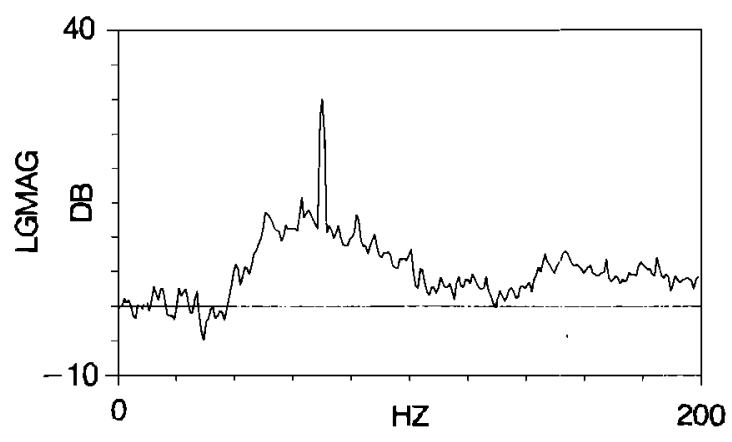

FIG. 6. Noise reduction for an input signal consisting of broadband noise from a centrifugal fan combined with a tone generated by a speaker ( $M=0.04-128$ averages) 
adaptive filter based on the RLMS algorithm. The effects of sound source and error path transfer functions are adaptively determined on line through the use of a second LMS algorithm that uses an independent low-level random noise source to model the sound source and error path while the system is operating. The combined system is fully adaptive, compensates for changes in all transducers, the source, and acoustical elements, is effective on broadband as well as narrow-band noise, and requires no calibration or training procedures prior to operation.

\section{ACKNOWLEDGMENTS}

The authors gratefully acknowledge the assistance of Cary Bremigan, James Gilbert, and Patricia Steaffens of the Nelson Industries, Inc. Corporate Research Department.

'L. J. Eriksson, "Active sound attenuation using adaptive digital signal processing techniques," Ph.D. thesis, University of Wisconsin-Madison (August 1985).

${ }^{2}$ L. J. Eriksson, M. C. Allie, and R. A. Greiner, "The selection and application of an IIR adaptive filter for use in active sound attention," IEEE Trans. Acoust. Speech Signal Process. ASSP.35 (4), 433-437 (1987).

${ }^{3}$ L. J. Eriksson and M. C. Allie, "A practical system for active attenuation in ducts," Sound Vib. 22(2), 30-34 (1988).

${ }^{4}$ L. J. Eriksson, M. C. Allie, C. D. Bremigan, and R. A. Greiner, "Active noise control using adaptive digital signal processing," Proc. IEEE ICASSP 88, New York, Vol. V, Paper A3.5, pp. 2594-2597.

${ }^{5}$ L. J. Eriksson and M. C. Allie, "System considerations for adaptive modelling applied to active noise control," Proc. IEEE ISCAS 88, Espoo, Finland, Vol. 3, pp. 2387-2390.

${ }^{6}$ L. J. Eriksson, "Active sound attenuation system with on-line adaptive feedback cancellation," U.S. Patent No. 4,677,677 (30 June 1987).
${ }^{7}$ P. L. Feintuch, "An adaptive recursive LMS filter," Proc. IEEE 64 (11), 1622-1624 (1976)

${ }^{8}$ B. Widrow, "Adaptive filters," in Aspects of Network and System Theory, edited by R. E. Kalman and N. Declaris (Holt, Rinehart, and Winston, New York, 1971).

${ }^{9} \mathrm{D}$. R. Morgan, "Analysis of multiple correlation cancellation loop with a filter in the auxiliary path," IEEE Trans. Acoust. Speech Signal Process. ASSP-28 (4), 454-467 (1980).

${ }^{10} \mathrm{~J}$. C. Burgess, "Active adaptive sound control in a duct: A computer simulation," J. Acoust. Soc. Am. 70, 715-726 (1981).

"B. Widrow, D. Shur, and S. Shaffer, "On adaptive inverse control," in Proceedings of the 15th Asilomar Conference on Circuits, Systems and Computers, 9-11 Nov. 1981, Pacific Grove, CA, pp. 185-189.

${ }^{12}$ B. Widrow and S. D. Stearns, Adaptive Signal Processing (Prentice-Hall, Englewood Cliffs, NJ, 1985), p. 288.

${ }^{13}$ L. A. Poole, G. E. Warnaka, and R. C. Cutter, "The implementation of digital filters using a modified Widrow-Hoff algorithm for the adaptive cancellation of acoustic noise," Proc. IEEE ICASSP 84, San Diego, Vol. 2, pp. 21.7.1-21.7.4.

${ }^{14}$ B. Widrow, J. M. McCool, and B. P. Medoff, "Adaptive control by inverse modeling," in Proceedings of the 12th Asilomar Conference on Circuits, Systems, and Computers, 6-8 November 1978, Pacific Grove, CA, pp. 90-94.

${ }^{15}$ L. J. Eriksson, "Active attenuation system with on-line modelling of speaker, error path, and feedback path," U.S. Patent No. 4,677,676 (30 June 1987)

${ }^{16}$ M. R. Schroeder, Number Theory in Science and Communications (Springer, Berlin, 1984).

${ }^{17}$ M. C. Allie, C. D. Bremigan, L. J. Eriksson, and R. A. Greiner, "Hardware and software considerations for active noise control," Proc. IEEE ICASSP 88, New York, Vol. V, Paper A3.6, pp. 2598-2601.

${ }^{18}$ Digisonix Division, Nelson Industries, Inc., "Digisonix digital sound cancellation systems for active noise control," Pub. No. DX-DS-1/987.

${ }^{19}$ L. J. Eriksson and M. C. Allie, "A digital sound control system for use in turbulent flows," Proceedings NOISE-CON 87, State College, PA, pp. 365-370.

${ }^{20}$ L. J. Eriksson, M. C. Allie, C. D. Bremigan, and J. A. Gilbert, "The use of active noise control for industrial fan noise," presented at the 1988 ASME Winter Annual Meeting, Chicago, IL, Paper 88-WA/NCA-4. 